\title{
Há equidade na produção do conhecimento sobre as doenças negligenciadas no Brasil?
}

There are equity promoting in the production of knowledge on neglected diseases in Brazil?

Hay equidad en la producción de conocimientos sobre las enfermedades olvidadas en Brasil?

Bruno Leonardo Alves de ANDRADE ${ }^{1}$

Dais Gonçalves ROCHA ${ }^{2}$

RESUMO: As doenças negligenciadas classificam-se como um grupo de doenças infecciosas fortemente associadas às condições de pobreza verificadas, em sua grande maioria, nos países periféricos. Dessa forma, elas são enquadradas como um clássico exemplo do déficit existente na produção do conhecimento destinado a determinados temas. Este artigo tem por objetivo apresentar as análises do atendimento das diretrizes de promoção da equidade na produção do conhecimento em doenças negligenciadas a partir do perfil dos pesquisadores e instituições com projetos aprovados pelos editais de pesquisa sobre o tema, publicados em 2006, 2008 e 2012 pelo Ministério da Saúde. Os dados investigados são públicos e foram coletados dos referidos projetos e da Plataforma Lattes/CNPq. O presente estudo de caráter transversal e descritivo obteve os seguintes resultados: 216 projetos contemplados nos três editais; a equivalência na participação do sexo masculino e feminino na coordenação das pesquisas; as instituições públicas de ensino superior e de pesquisa foram as mais contempladas pelos editais; a maior parte dos estudos foi proveniente de autores com formação na área das ciências da saúde e concentrou-se principalmente nas instituições situadas na região Sudeste, que vale destacar, não é a região do país que concentra o maior número de casos das doenças negligenciadas.

Palavras-chaves: Doenças negligenciadas. Doenças tropicais negligenciadas. Equidade.

ABSTRACT: Neglected diseases are classified as an infectious disease group that is strongly associated to poverty conditions, mostly in peripheral countries. From this perspective, neglected diseases are shown as a classic example of science production deficits in certain research topics. This article presents the analyses of the promotion of equity within knowledge production about neglected diseases based on researcher profiles, institutions with projects approved on the subject, and published in 2006, 2008, and 2012 by the Brazilian Ministry of Health. The data investigated are public and collected from these projects and the Lattes Platform/CNPq. This cross-sectional descriptive study obtained the following results: 216 projects included in the three calls for application; almost equal gender participation in coordinating research;

\footnotetext{
1 Mestre em Odontologia com área de concentração em Saúde Coletiva pela Faculdade de Odontologia da Universidade de Pernambuco e Doutor em Bioética pela Universidade de Brasília.

2 Graduação em Odontologia- UFG (1990), mestrado (1997) e doutorado (2001) em Saúde Pública-USP. PósDoutorado na University of British Columbia/ School of Population and Public Health- Canadá. Professora do Depart. Saúde Coletiva-UnB. Integrante do GT de Promoção da Saúde e DLIS. 
predominantly developed by mostly public higher education institutions (universities) and research centers. Most of the studies were conducted by authors trained in health sciences and were mainly concentrated in institutions of the southeastern region of Brazil, which is notably not the part of the country with the largest number of neglected disease cases. Key-words: Neglected diseases. Neglected tropical diseases. Equity.

RESUMEN: Las enfermedades olvidadas son clasificados como un grupo de enfermedades infecciosas fuertemente asociados con la pobreza marcada, sobre todo en los países periféricos. Por lo tanto, se clasifican como un ejemplo clásico del déficit existente en la producción de conocimiento para ciertos temas. Este artículo tiene como objetivo analizar el cuidado del patrimonio directrices de promoción en la producción de conocimientos sobre las enfermedades olvidadas desde el perfil de los investigadores e instituciones con proyectos aprobados por los edictos de investigación sobre el tema, publicado en 2006, 2008 y 2012 por el Ministerio de Salud. Los datos son públicos y se investigó se obtuvieron de estos proyectos y la Plataforma Lattes/CNPq. Este estudio descriptivo transversal obtuvo los siguientes resultados: 216 proyectos incluidos en los tres avisos; la equivalencia en la participación de hombres y mujeres en la coordinación de la investigación; instituciones públicas de educación superior y la investigación fueron los más cubiertos por los anuncios; la mayoría de los estudios fueron de autores formados en las ciencias de la salud y en especial por la concentración en instituciones ubicadas en el sureste, que es digno de mención, no es del país que tiene el mayor número de casos de enfermedades olvidadas.

Palavras-clave: Enfermidades olvidadas; Enfermidades tropicales olvidadas; Equidad.

\section{INTRODUÇÃO}

Há tempos a humanidade é assolada por um grupo de doenças que estão associadas à pobreza e proliferam, principalmente, em ambientes marcados pela exclusão social. Essas doenças são denominadas doenças negligenciadas e de acordo com a Organização Mundial da Saúde (OMS) formam um conjunto de 17 enfermidades que afetam principalmente pessoas que vivem nos trópicos, mas não são exclusivas a eles ${ }^{(1)}$.

Nessa relação estão incluídas as seguintes enfermidades: tracoma; úlcera de Buruli; tripanossomose africana (doença do sono); doença de Chagas (tripanossomíase americana); dengue; dracunculíase; cisticercose; leishmaniose; hanseníase; filariose linfática; oncocercose; esquistossomose; bouba; geohelmintíase; raiva; equinococose e fasciolíase ${ }^{(1)}$.

Dados da OMS ${ }^{(1)}$ sobre o tema apontam que muitas delas já foram altamente prevalentes, mas, na medida em que as sociedades se desenvolveram e as condições de vida melhoraram foram desaparecendo gradualmente.

A negligência que permeia o histórico de determinadas doenças transmissíveis associadas à pobreza impacta diretamente nas condições sociais e econômicas de populações marginalizadas 
concentradas, na sua grande maioria, nas áreas pobres de países de baixa e média renda. Hoje, mesmo com o premente risco de contaminação de pelo menos $40 \%$ da população mundial por uma doença negligenciada, verifica-se que elas permanecem na sua grande maioria ocultas, concentradas em áreas rurais remotas ou em favelas urbanas ${ }^{(2)}$.

De fato, dentre os países da América Latina e Caribe, o Brasil é o país que apresenta o maior número de casos de doenças negligenciadas. Desta forma, grandes partes dos milhares de brasileiros que se encontram em situação de pobreza estão ou serão contaminados por uma ou mais de uma dessas doenças ${ }^{(3)}$.

O breve quadro apontado pelos autores deste artigo reforça que as iniquidades e as injustiças sanitárias que acometem, preferencialmente, os países periféricos persistem, na maioria das vezes, quando não são adotados mecanismos de enfrentamento, principalmente, por parte das autoridades sanitárias.

No Caso do Brasil, apesar da tendência a estabilidade verificada para este grupo de doenças ${ }^{(4)}$, um olhar ético e ações governamentais que estimulem as pesquisas e suas consequentes aplicações devem ser estimuladas e direcionadas por uma justa divisão de recursos, sobretudo, para as regiões do país com menor estrutura para produção científica.

Para os autores deste artigo, ações deste tipo são corroboradas, sobretudo, quando se encara a pesquisa como um investimento e não como uma despesa. Pois, em se tratando de pesquisa em saúde, ela é essencial para elucidação da natureza e do contexto dos problemas, para desenvolvimento e implementação de intervenções que salvam vidas, na busca de melhorias para os determinantes sociais da saúde e consequentemente na promoção da celeridade no acesso aos benefícios, principalmente, para as populações pobres e marginalizadas que se encontram a margem do processo científico e tecnológico.

O que se observa é que a deficiência no processo de estabelecimento e de execução de prioridades no processo de produção do conhecimento em saúde ainda é um fato presente, apesar de já ter sido demonstrado no final da década de 90 pela Comissão de Pesquisa em Saúde para o Desenvolvimento ${ }^{(5)}$ quando apontou a existência de uma dissociação entre carga de doenças das populações desfavorecidas e a concentração de investimento global em pesquisa em saúde nos problemas do mundo industrializado.

De fato, existe um desajuste onde menos de $10 \%$ dos investimentos de recursos destinados para pesquisa e desenvolvimento em saúde são direcionados às doenças ou condições que atingem $90 \%$ da população mundial, determinando um desequilíbrio que foi batizado de déficit 10/90 - The 10/90 Gap $^{(5)}$.

Desta forma, as doenças negligenciadas são consideradas como um clássico exemplo desse desajuste. Vive-se a sombra da contradição. Enquanto milhares de pessoas necessitam urgentemente Tempus actas de saúde colet. Brasília, 9(3), 21-34, set, 2015.

ISSN 1982-8829 
de medidas de intervenção para combater doenças relacionadas às suas precárias condições de vida, a produção do conhecimento, mais especificamente a pesquisa e desenvolvimento (P\&D) em saúde, desenvolvida principalmente pela indústria farmacêutica mundial, volta sua atenção para as doenças globais, sempre na intenção de produzir e comercializar para obter lucros.

Dito isto, a produção do conhecimento no país necessita do direcionamento para áreas prioritárias e deve ser concebida à luz dos referenciais da equidade para promover tratamento diferente para os diferentes, promovendo uma justa distribuição desigual com a menor exclusão possível das partes envolvidas ${ }^{(6)}$.

Nesse sentido, no ano de 2003 foi iniciado no país pelo Ministério da Saúde (MS) um processo de indução a produção de conhecimento destinado às doenças relacionadas à pobreza denominadas por doenças negligenciadas. Inicialmente foram lançados editais por doenças específicas (tuberculose 2003; dengue 2004 e hanseníase 2005), sendo em seguida lançados três editais (2006, 2008 e 2012) para doenças consideradas negligenciadas e prioritárias (dengue, doença de Chagas, esquistossomose, hanseníase, leishmanioses, malária, tuberculose, tracoma e geohelmintíases) na visão dos gestores, pesquisadores e profissionais da área da saúde ${ }^{(7,8)}$.

Face ao exposto, os autores do presente artigo se propõem a analisar o atendimento das diretrizes de promoção da equidade na produção do conhecimento sobre o tema das doenças negligenciadas, a partir do apoio dos editais para as pesquisas da área, publicados no país nos anos de 2006, 2008 e 2012.

\section{METODOLOGIA}

O presente estudo foi de caráter transversal e descritivo. Para obtenção dos resultados foram utilizados dados dos projetos de pesquisas aprovados pelos editais de fomento à pesquisa e desenvolvimento na área das doenças negligenciadas lançados pelo Ministério da Saúde e parceiros nos anos de 2006, 2008 e 2012. A escolha dos editais ocorreu, sobretudo, pela sua expressividade no cenário da pesquisa em saúde do Brasil.

Os dados que compuseram são públicos e se encontram disponíveis no sítio eletrônico www. pesquisasaude.saude.gov.br e na Plataforma Lattes/CNPq. A consulta à base de dados foi realizada entre os meses de novembro e dezembro de 2012 e complementada no mês dezembro de 2014.

Para a construção do perfil dos pesquisadores foram consideradas as seguintes variáveis: sexo e formação dos coordenadores das pesquisas, tipo de instituição as quais estão vinculados (caráter e atuação) e região de localização no país dessas instituições.

O levantamento bibliográfico iniciou-se em janeiro de 2012 e foi finalizado em dezembro de 2014. A busca ocorreu na área da "Ciências da Saúde em Geral" e a fonte de consulta foi a Biblioteca Virtual em Saúde (BVS), cuja captação reúne as bases LILACS, IBECS, MEDLINE, Biblioteca Cochrane e SciELO. 
Foram utilizados para consulta os seguintes descritores: doenças negligenciadas, doenças tropicais negligenciadas, produção cientifica e pesquisa e desenvolvimento em saúde, e neglected diseases, neglected tropical diseases, scientific production, research priorities, health research and development.

Por fim, os autores do estudo destacam que este artigo trata-se do primeiro produto da tese intitulada de $A$ produção do conhecimento em doenças negligenciadas no Brasil: uma análise bioética dos dispositivos normativos e da atuação dos pesquisadores brasileiros e que a submissão e aprovação do projeto desta tese ocorreu em outubro de 2012 pelo Comitê de Ética em Pesquisa da Faculdade de Ciências da Saúde da Universidade de Brasília (CEP FS-UnB) sob o número 142/12.

\section{RESULTADOS}

O universo de pesquisadores contemplados nos editais de fomento para pesquisa e desenvolvimento para as doenças negligenciadas, lançado pelo Ministério da Saúde e parceiros nos anos de 2006, 2008 e 2012 foi de 216 pesquisadores. Em 2006, 47(58\%) dos contemplados eram do sexo masculino e $35(42 \%)$ do sexo feminino gerando um total de 82 pesquisadores para aquele ano. No ano de 2008, o sexo masculino apresentou 29 contemplados (50\%) e o sexo feminino também apresentou 29(50\%) contempladas, com um total de 58 pesquisadores. Já em 2012, foram 38 do sexo feminino e 38 do sexo masculino.

Em relação à formação dos pesquisadores, no ano de 2006 foram observados os seguintes resultados: 67(82\%) pesquisadores foram formados na área das ciências da saúde, 14(17\%) pesquisadores das ciências exatas e 1(1\%) pesquisador não apresentou registro do seu currículo na Plataforma Lattes para verificação. Para o ano de 2008 foi observado que 48(83\%) dos pesquisadores eram das ciências da saúde, $8(14 \%)$ pesquisadores tinham formação na área das ciências exatas e $2(3 \%)$ pesquisadores não tinham seu currículo registrado na Plataforma Lattes. Para o ano de 2012, 74(97\%) pesquisadores tinham formação na área das ciências da saúde e 2(3\%) pesquisadores apresentaram formação na área das ciências humana. Tratando-se da formação de pós-graduação, do universo de 216 pesquisadores contemplados nos três editais, $212(98 \%)$ apresentaram o título de doutor, $1(0,6 \%)$ de especialista e 3(1,4\%) não tinham registro dos seus currículos na Plataforma Lattes.

Os autores verificaram que as instituições contempladas pelos editais eram as de ensino superior, os institutos de pesquisa, os hospitais de ensino, os serviços de saúde e as organizações sem fins lucrativos (ONG, OSCIP, etc). No ano de 2006 (Tabela 1), a contemplação ocorreu da seguinte forma: 50(61\%) instituições de ensino superior, 31(38\%) institutos de pesquisa e 1(1\%) serviço de saúde. 
Tabela 1 - Distribuição das instituições contempladas no Edital de apoio à pesquisa para doenças negligenciadas por regiões do país no ano de 2006

\begin{tabular}{lccccccc}
\hline & \multicolumn{7}{c}{ Região } \\
\cline { 3 - 8 } & \multicolumn{1}{c}{ Tipo de Instituição } & SE & NE & CO & S & N & Total \\
Instituto de Pesquisa & 23 & 2 & 1 & 2 & 3 & 31 \\
Ensino Superior & 30 & 12 & 0 & 7 & 1 & 50 \\
Hospital de ensino & 0 & 0 & 0 & 0 & 0 & 0 \\
Serviço de Saúde & 1 & 0 & 0 & 0 & 0 & 1 \\
Outras organizações sem fins lucrativos (ONG, OSCIP etc.) & 0 & 0 & 0 & 0 & 0 & 0 \\
\hline Total & $\mathbf{5 4}$ & $\mathbf{1 5}$ & $\mathbf{1}$ & $\mathbf{9}$ & $\mathbf{3}$ & $\mathbf{8 2}$ \\
\hline
\end{tabular}

Fonte: MS/SCTIE/DECIT

Em 2008 (Tabela 2) foram contempladas as seguintes instituições: 39(67\%) instituições de ensino superior, $16(27,5 \%)$ institutos de pesquisa, 2(3,5\%) hospitais de ensino e $1(2 \%)$ organização sem fins lucrativos.

Tabela 2 - Distribuição das instituições contempladas no Edital de fomento à pesquisa para doenças negligenciadas por regiões do país no ano de 2008

\begin{tabular}{|c|c|c|c|c|c|c|}
\hline \multirow[b]{2}{*}{ Tipo de Instituição } & \multicolumn{6}{|c|}{ Região } \\
\hline & SE & NE & CO & $\mathbf{S}$ & $\mathbf{N}$ & Total \\
\hline Instituto de Pesquisa & 15 & 1 & 0 & 0 & 0 & 16 \\
\hline Ensino Superior & 20 & 9 & 2 & 4 & 4 & 39 \\
\hline Hospital de ensino & 2 & 0 & 0 & 0 & 0 & 2 \\
\hline Outras organizações sem fins lucrativos (ONG, OSCIP etc.) & 1 & 0 & 0 & 0 & 0 & 1 \\
\hline Total & 38 & 10 & 2 & 4 & 4 & 58 \\
\hline
\end{tabular}

Fonte: MS/SCTIE/DECIT

No ano de 2012 (Tabela 3) foram 51(67\%) instituições de ensino superior, 24(31,7\%) institutos de pesquisa e 1(1,3\%) serviço de saúde. Vale destacar que a maioria das contemplações nos três editais ocorreu para instituições de caráter público. Além disso, a região Sudeste do país foi a região com o maior número de instituições contempladas, seguida pela região Nordeste do Brasil (Tabelas 1, 2 e 3).

Tabela 3 - Distribuição das instituições contempladas no Edital de apoio à pesquisa para doenças negligenciadas por regiões do país no ano de 2012.

\begin{tabular}{|c|c|c|c|c|c|c|}
\hline \multirow[b]{2}{*}{ Tipo de Instituição } & \multicolumn{6}{|c|}{ Região } \\
\hline & SE & $\mathbf{N E}$ & $\mathrm{CO}$ & $\mathbf{S}$ & $\mathbf{N}$ & Total \\
\hline Instituto de Pesquisa & 17 & 3 & 0 & 0 & 4 & 24 \\
\hline Ensino Superior & 26 & 12 & 3 & 8 & 2 & 51 \\
\hline Hospital de ensino & 0 & 0 & 0 & 0 & 0 & 0 \\
\hline Serviço de Saúde & 0 & 0 & 0 & 0 & 1 & 1 \\
\hline Outras organizações sem fins lucrativos (ONG, OSCIP etc.) & 0 & 0 & 0 & 0 & 0 & 0 \\
\hline Total & 43 & 15 & 3 & 8 & 7 & 76 \\
\hline
\end{tabular}

Fonte: MS/SCTIE/DECIT 


\section{DISCUSSÃO}

O modo de produção do conhecimento na área da saúde do país atravessa um período de transformações significativas, em que as verdadeiras necessidades da sociedade deixam de ser latentes para se tornarem explícitas e assumirem o lugar de destaque que lhe é devido dentre as ações de responsabilidade do Estado.

A recorrência dos editais públicos de apoio às pesquisas das doenças negligenciadas tem propiciado um cenário favorável para a produção do conhecimento sobre o tema no Brasil. Profundas transformações no modo nacional de produção do conhecimento e a relação estabelecida entre ciência e sociedade, que concedem uma maior integração entre necessidades sociais e pesquisa científica outrora afirmada por Pellegrini (9), começam a refletir na totalidade do processo de apoio à pesquisa em saúde em andamento no país.

De fato, apesar de não estarmos no mesmo patamar dos países de alta renda, considerados como países centrais, existe o empenho para que as deficiências presentes na situação da pesquisa do país sejam superadas e, cada vez mais, o estímulo à produção do conhecimento acerca de temas que refletem diretamente nas condições de desenvolvimento de uma nação, como no caso do tema das doenças negligenciadas, ganhe mais espaço nas agendas de pesquisa e tornem-se prioritários.

As evidencias apontam que as ações governamentais voltadas para a pesquisa de doenças enquadradas no status de negligência são uma prática verificada no país, a exemplo dos editais publicados nos anos de 2003(tuberculose), 2004 (dengue) e 2005(hanseníase).

No entanto, o presente estudo constatou que nenhuma ação de apoio à pesquisa tenha sido tão expressiva quanto as promovidas nos anos de 2006, 2008 e 2012. Nenhum edital, até então, tinha contemplado tantas linhas de pesquisa para um número tão expressivo de doenças (trouxe sete doenças como prioridade no conteúdo dos editais de 2006 e 2008 e nove doenças no edital de 2012). De acordo com o Departamento de Ciência e Tecnologia do Ministério da Saúde DECIT/ MS (10), o edital temático das doenças negligenciadas foi considerado como a mais importante iniciativa do ano de 2006, tanto pela relevância do tema quanto ao aporte de recursos destinado para o edital.

Nesse sentido, verifica-se que em termos de financiamento público, o Brasil está em sexto lugar no ranking dos países que mais investem no segmento das doenças negligenciadas, e o primeiro, se considerarmos apenas os países em desenvolvimento onde essas doenças são endêmicas (11).

Atualmente, as medidas adotadas pelo país relativas à pesquisa em doenças negligenciadas, refletem na sua liderança mundial em pesquisas sobre o tema (12), demonstrando, inclusive, a supressão do abismo científico (13) instituído no país entre a área das doenças negligenciadas com outras áreas da saúde.

Além de investir de modo expressivo em pesquisas sobre o tema, o país também passou a 
investir na produção de insumos utilizados no combate e no tratamento de determinadas doenças negligenciadas, a exemplo do medicamento Benzonidazol utilizado no combate da doença de Chagas e dos medicamentos para tuberculose (12).

As evidências científicas confirmam que a vinculação de programas de combate a doenças, como nos casos dos bem sucedidos programas de controle da varíola, oncocercose e doença de Chagas $(14,15)$ devem, em parte, seu sucesso ao fato de terem incluído como parte das suas ações atividades de pesquisa, reforçando a ideia que a produção do conhecimento científico torna-se um forte componente para superação das iniquidades na medida em que integram as verdadeiras necessidades da sociedade a agenda de pesquisa vigente no país.

Nesse sentido, Sanders et al (16) reforçam que as ações de saúde, como no caso das pesquisas, devem ter seu ordenamento baseado tanto na crescente dívida existente entre localidades ricas e pobres, como também no espaço existente entre a disponibilidade do conhecimento científico e sua efetiva aplicação para solucionar problemas de saúde que acometem principalmente as populações expostas à pobreza.

A louvável tentativa do país em estabelecer uma nova ordem no campo da pesquisa em saúde, além de considerar as necessidades prementes na realidade dos coletivos socialmente excluídos, considerou também as diferenças regionais presentes nas cinco regiões do país em que as pesquisas foram produzidas. Como consequência, foi prescrita uma medida pelos editais na intenção de abrandar tais diferenças e incentivar o acesso dos estudos provenientes das regiões menos estruturadas e com altos índices de doenças negligenciadas.

Houve um incentivo pautado no combate das iniquidades regionais da pesquisa foi instituído para todas as regiões do país, inclusive, para as regiões com maior prevalência das doenças negligenciadas e com menor capacidade para pesquisa, como é o caso das regiões geográficas do Norte, Nordeste e Centro-Oeste (13).

$\mathrm{Na}$ visão de Morel et al (13), esse tipo de incentivo funciona para estimular a produção do conhecimento em regiões aonde às iniquidades regionais no campo da pesquisa em saúde são visíveis e inversamente proporcionais a prevalência de muitas doenças. No entanto, constatou-se que a maioria das pesquisas contempladas com recursos públicos foram os estudos realizados por instituições de pesquisas da região mais desenvolvida, com melhor estrutura e que não se enquadra na maior prevalência de casos de doenças negligenciadas no país, no caso, a região Sudeste.

A realidade apontada por esta pesquisa não foge aos padrões da situação da produção do conhecimento vigente no país. Quando se analisa a produção relativa ao tema das doenças negligenciadas, verifica-se que esta ainda se concentra, principalmente, nas instituições situadas no Rio de Janeiro e São Paulo, estados da região Sudeste $(17,4,18)$, seguidas pela produção das regiões Nordeste, Norte e Centro-Oeste. 
Os dados analisados confirmam que o apoio público à produção científica nacional acerca do tema das doenças negligenciadas além de ascendente é um dos mais expressivos da área da saúde (12). Entretanto, nem todos os grupos de pesquisas conseguem desfrutar de tal apoio. Percebe-se que os grupos de pesquisa mais estruturados, localizados na sua grande maioria em instituições de pesquisas situadas na Região Sudeste do país, detêm o maior poder de captação dos recursos públicos para o desenvolvimento dos seus estudos.

Apesar da tentativa de fortalecimento da capacidade de pesquisa pelo apoio público das regiões com maiores necessidades, como no caso do Norte, Nordeste e Centro-Oeste, verifica-se ainda que o crescimento da produção científica voltada às doenças negligenciadas nessas regiões é aquém da que ocorre na região de maior desenvolvimento e capacidade de captação de recursos do país.

O que se verificou entra as regiões foi uma divisão bastante desigual e ainda pouco articulada, permanecendo algumas dificuldades de cooperação interinstitucional para formação de redes nacionais e regionais de pesquisa que integrem as de maior densidade tecnológica e produtividade com as regiões de menor densidade e menos produtivas (19).

A despeito das investidas do Estado, constatou-se que uma repartição injusta e ineficaz dos recursos destinados à área ainda é algo que deixa o Norte, Nordeste e Centro-oeste do país, que são regiões que apresentam alta prevalência de doenças negligenciadas $(13,18)$, num certo descompasso do processo vigente no país de incentivo a produção conhecimento da área.

Apesar da tentativa de se estabelecer um parâmetro que considere a equidade como ponto de partida para divisão dos projetos em doenças negligenciadas por região do país, poucas modificações foram observadas no cenário da pesquisa acerca deste tema. Com isso, verifica-se que o descumprimento do critério de equidade estabelecido pelas normativas foi algo presente. A quantidade de projetos contemplados das Regiões Norte, Nordeste e Centro-Oeste, além de não representarem nem a metade dos projetos contemplados pela Região Sudeste, não chegou ao patamar estabelecido pelas normativas vigente para a pesquisa em saúde no país.

O que se pôde observar foi que as instituições que ocupam lugar de destaque no ranking de produção do conhecimento foram às mesmas contempladas pelos dois editais, concentradas em sua grande maioria nos estados de São Paulo e Rio de Janeiro. Deste modo, há uma sugestão que as mesmas instituições perpetuam o seu poder e suas subjetividades, ferem o que se considera por equidade nas ações de saúde e confirmam a máxima de quem tem o conhecimento, detém o poder.

No momento da contemplação de instituições de pesquisa, critérios de igualdade não devem ser considerados, e sim a diferença das condições existentes, levando-se em conta as necessidades diferenciadas. Assim, uma ação que pretende ser guiada pela equidade deveria proporcionar à satisfação não apenas aos mais fortes e, sim, as necessidades de todos os envolvidos.

Nesse caso, critérios de equidade deveriam prevalecer, pois, além de respeitar as diferenças, 
poderia ser um caminho para que se atingisse a igualdade das partes envolvidas. Corroborando com a visão dos autores deste estudo, Fortes (20) afirmou que a equidade pode ser considerada como"a cada um conforme as suas necessidades". Nunes (21), por sua vez, complementou tal visão, pois afirma que a eleição dos mais necessitados é central na elaboração de qualquer metodologia que pretenda alocar ou alterar alocação de recursos, que no primeiro momento, para ser mais equitativa, a alocação deverá ocorrer pela seleção de necessidades.

Apesar da distribuição não equitativa dos projetos por regiões, o incentivo do Poder Público à produção do conhecimento voltada ao combate das doenças negligenciadas pode atuar como uma ação cuja consequência possibilita a promoção de bem estar para um maior número de pessoas, principalmente, para indivíduos e os coletivos que são socialmente excluídos.

O processo de produção do conhecimento estimulado pelos editais de 2006, 2008 e 2012 foi exercido, na sua grande maioria, pelas instituições universitárias, consideradas o lócus privilegiado de construção científica e de desenvolvimento tecnológico, não só no Brasil, mas em todo mundo (22), foi corroborado pelas evidências apontadas por Iozi (23) ao afirmar que as atividades de pesquisa no Brasil ocorrem em instituições universitárias e em instituições de pesquisa específicas da área da saúde, com uma incipiente participação das unidades de serviço, com exceção dos hospitais de ensino, e das empresas privadas do setor.

Seguindo esta tendência, as universidades e centros de pesquisas da Região Sudeste do país mantiveram-se como o locus preferencial da produção do conhecimento em doenças negligenciadas no país ao longo da série temporal analisada por este estudo. Leta et al (24) já haviam demonstrado esta dinâmica ao afirmar que a produção de pesquisa do país estaria concentrada nas universidades e em alguns centros de pesquisa da região mais desenvolvida do país.

Desta forma, os fatos acima apontados confirmam que o ciclo virtuoso, instituído principalmente pelas universidades públicas desde meados dos anos 60, destinado a formar e consolidar grupos de pesquisa (25), passou a ter um comportamento de "ciclo vicioso", explicitado pela tendência da supremacia e das subjetividades de muitas instituições e de seus pesquisadores na perpetuação e consequente reforço das desigualdades regionais na produção do conhecimento verificada no cenário da pesquisa em saúde no país.

Os resultados relativos à localização geográfica e tipos de instituições contempladas pelos editais anteriormente apontados pelos autores, demonstram que a situação da pesquisa destinada às doenças negligenciadas no país está em consonância com a situação das demais pesquisas realizadas pela área da saúde, ou seja, pesquisas realizadas por instituições públicas e com o apoio público na maioria dos casos.

De acordo com os dados apontados pela Secretaria de Ciência, Tecnologia e Insumos Estratégicos do Ministério da Saúde (26), os grupos que realizam atividades de pesquisa em saúde no país, 49\% estão localizados na região Sudeste, $23 \%$ na região Sul, $17 \%$ na região Nordeste, $6 \%$ na região Centro-Oeste e $5 \%$ na região Norte. 
Em relação ao sexo, os resultados demonstraram um equilíbrio desta variável para os três editais e corroboraram com as afirmações de Minayo (22) quando apontou que à área de Ciência, Tecnologia e Inovação (C\&T\&I) tem apresentado um caminho positivo quanto à igualdade de participação de homens e mulheres no cenário nacional da pesquisa científica. De acordo com esta autora, em 2010 a participação percentual das mulheres no setor pareou com a dos homens, tendo havido também crescimento contínuo na condição de liderança de grupos de pesquisa. Em 2010 a situação da pesquisa era a seguinte: $54,9 \%$ de homens líderes de pesquisa e $45,1 \%$ de mulheres líderes de pesquisa; contra 66,8\% e 33,2\%, respectivamente para o ano de 2000.

\section{CONSIDERAÇÕES FINAIS}

Diante do exposto, verifica-se que a indução à pesquisa em doenças negligenciadas, quando colocada em prática, não pode ser direcionado e ficar restrito apenas aos centros de pesquisa situados nas instituições de maior projeção do país. Os centros de pesquisas do país que trazem nos seus históricos a aptidão para a realização de estudos de relevância para a temática devem introjetar nas suas práticas mecanismos capazes de aproximá-los das instituições que, apesar de capazes, apresentam-se com menor aptidão para o desenvolvimento das pesquisas. Ou seja, devese partir de uma lógica de aptidão institucional para a lógica da capacidade de quem quer produzir conhecimento científico no país.

Destaca-se que, apesar de ser considerada como um dos principais meios de enfrentamento para o status de negligência condicionado a determinadas doenças infecciosas, a pesquisa científica não é o único meio. Portanto, uma abordagem mais ampla se faz necessária, principalmente, pela complexidade que envolve o tema. Visões que não se prendam apenas as questões atinentes à assistência à saúde devem ser incorporadas para esta temática, pois, apesar de se tratar do enfrentamento a uma entidade nosológica, não se pode aprisionar o tema apenas à lente da causalidade biológica que o envolve, da sua ocorrência individualizada e dissociada dos fenômenos sociais que o cerca.

É impossível estabelecer uma dissociação da relação entre doença e doente, doente e seu meio social, fazendo-se necessária um olhar convergente dos vários tipos de pesquisas (biomédicas, clínica, desenvolvimento tecnológico, saúde coletiva e ciências sociais e humanas) para o tema. Além de existir doenças negligenciadas, existem também indivíduos/coletivos (doentes) negligenciados ou indivíduos/coletivos que apesar de não estarem doentes, caso não haja algum tipo de intervenção, serão enquadrados como possíveis suscetíveis ao acometimento de uma ou de várias doenças negligenciadas.

O grande desafio lançado ao país no campo da produção do conhecimento destinado as doenças negligenciadas é justamente a construção da relação entre as ações de estímulo destinadas ao fortalecimento da capacidade de produção científica voltada ao tema e o estabelecimento de formas equitativas de alocação dos recursos que se destinam a este tipo de produção. 
De fato, quando adequadamente conduzidas, as ações podem tanto potencializar a produção científica da área no país, inclusive de centros de pesquisa das regiões menos desenvolvidas como o Norte, Nordeste e Centro-Oeste, como também ser revertida em melhorias para saúde da população em geral.

Considerou-se que a produção do conhecimento deve ser sinérgica e estar em consonância com as verdadeiras necessidades da sociedade, fazendo com que seja estabelecida uma estreita e verdadeira relação entre pesquisa, política de saúde e equidade.

Há indicativo para que outros estudos, quer sejam de caráter analítico ou prospectivo, produzidos pela área da saúde ou por outras áreas do conhecimento, sejam concebidos para possibilitar o aprofundamento acerca do tema da produção do conhecimento em doenças negligenciadas no país

Por fim, recomenda-se aprofundar a compreensão e utilização de formas para diminuir a distância estabelecida entre a produção do conhecimento/resultados da pesquisa e a sua aplicabilidade como elemento norteador tanto na construção de políticas públicas quanto nas ações voltadas para atenção à saúde.

\section{REFERÊNCIAS BIBLIOGRÁFICAS}

1. WHO. First Who report neglected tropical diseases: working to overcome the global impact neglected tropical diseases. WHO/HTMA/NTD/2010.1. Geneve. Disponível em < http:// www.who.int/neglected_diseases/2010report/en/> Acesso em: 10 jun. 2013.

2. Batalha E, Morosini L. Atenção aos esquecidos. Radis. 2013,124:8-17; Disponível em <

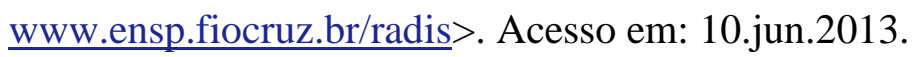

3. Hotez, P. The giant anteater in the room: Brazil's neglected tropical diseases problem. PLoS Neglected Tropical Diseases. 2(1):77. 2008.

4. Noronha, J.S., Silva, T.R., Szklo, F., Barata, R.B. O que os pesquisadores pensam do sistema de pesquisa em saúde no Brasil: um estudo piloto. RECIIS - R. Eletr. De Com. Inf. Inov. Saúde. Rio de Janeiro, 2012, 6 (1): 13-25.

5. Global Forum for Health Research 2002. The 10/90 Report on Health Research 2001-2002. GFHR; 2001.

6. Siqueira-Batista, R.; Schramm, F. R. A saúde entre a iniquidade e a justiça: contribuições da igualdade complexa de Amartya Sen. Ciência \& Saúde Coletiva, Rio de Janeiro, 2005. (1):129142.

7. Brasil. Ministério da Saúde. Oficina de Prioridades de Pesquisas em Saúde de Doenças Negligenciadas. Informativo DECIT - Departamento de Ciência e Tecnologia/SVS da Secretaria de Vigilância em Saúde do Ministério da Saúde. Julho, 2008. 
8. Brasil. Ministério da Saúde. Departamento de Ciência e Tecnologia. Prioridade de Pesquisa em Saúde. Caderno 2 - Doenças Negligenciadas. Departamento de Ciência e Tecnologia - Decit 2006. Disponível em:<http://bvsms.saude.gov.br/bvs/publicacoes/prioridades_invest/i2_tela4_2. html> Acesso em 4 abr 2012.

9. Pellegrini Filho, A. Pesquisa em Saúde, política de saúde e equidade na América Latina. Ciência Saúde \& Coletiva, Rio de Janeiro, 2004, 9(2): 339-350.

10. Brasil. Ministério da Saúde. Secretaria de Ciência, Tecnologia e Insumos Estratégicos. Departamento de Ciência e Tecnologia. Pesquisa em Saúde no Brasil. Rev. Saúde Coletiva, 42(4):773-5; 2008.

11. Assad, L. Doenças negligenciadas estão nos países pobres e em desenvolvimento. Ciência e Cultura, São Paulo, 2010, 62 (1): 6-8.

12. Sociedade Brasileira de Medicina Tropical. Ministério da Saúde afirma que enfrentamento das doenças negligenciadas é prioridade. 2012. Disponível em < http://www.sbmt.org.br/site/ corpo_texto/187> Acesso em: 08 out. 2014.

13. Morel, C.M., Serruya S.J., Penna G.O., Guimarães R. Co-authorship Network Analysis: A Powerful Too for Strategic Planning of Research, Development and Capacity Building Program on Neglected Diseases. PLoS Negl Trop Dis. Switzerland, ago 2009, 3(8): e501.

14. Molyneux, DH; Morel C. Onchocerciasis and Chagas disease control: the evolution of control via aplied research through changing development scenarios. British Medical Bulletin; 1998, 54(2): 327-339.

15. Henderson, DA. Eradication: lessons from the pat. Bull. World Health Organ 1998, 76 (Suppl 2): 17-21.

16. Sanders, D. et al. Making research matter: a civil society perspective on health research. Bulletin of the World Heakth Organization, 2004, 82 (10):757-763.

17. Cunha Santos, M.S., Santos, Lima L., Martelli, D.R.B., Martelli - Júnior, H. Perfil dos pesquisadores da Saúde Coletiva no Conselho Nacional de Desenvolvimento Científico e Tecnológico. Physis Revista de Saúde Coletiva, Rio de Janeiro, 2009, 19(3): 761 - 775.

18. Brasil. Ministério da Saúde. Secretaria de Vigilância em Saúde. Departamento de Análise de Situação em Saúde. Saúde Brasil 2013: uma análise da situação de saúde e das doenças transmissíveis relacionadas à pobreza. Brasília: Ministério da Saúde, 2014: 384.

19. Cavalcante, LR. Desigualdades regionais em Ciência, Tecnologia e Inovação (CT\&I) no Brasil: uma análise de sua evolução recente. Rio de Janeiro: Instituto de Pesquisa Econômica Aplicada - Ipea, texto para discussão (1574), 2011. 
20. Fortes, PAC. Reflexão bioética sobre priorização e o racionamento de cuidados de saúde: entre a utilidade social e a equidade. Cad. Saúde Pública, Rio de Janeiro, 2008, 24(x): 1-6

21. Nunes, A. Alocação equitativa inter-regional de recursos públicos federais do SUS: a receita própria do município como variável moderadora. Brasília: Sistema de Informações sobre Orçamentos Públicos em Saúde, Departamento de Economia da Saúde, Secretaria de Ciência, Tecnologia e Insumos Estratégicos, Ministério da Saúde; 2004.

22. Minayo, M.C.S. Global \& Local, Meritocrático \& Social: O papel da Ciência e Tecnologia em Saúde Coletiva no Brasil. Revista Tempus Actas de Saúde Coletiva. 2012, 6 (2).

23. Iozzi, FL. Ciência, tecnologia e inovação no Brasil: panorama atual das políticas federais para a saúde. In: Viana ALD, Ibañez N, Bousquat A. Saúde, desenvolvimento, ciência, tecnologia e inovação. São Paulo: HUCITEC-Cealag, 2012:150-173.

24. Leta, J. Glanzel; Thus, B. Science in Brazil. Part 2: sectoral and institutional research profiles. Scientometrics, 2006, 67(1): 87-105.

25. Guimarães, JA. A pesquisa médica e biomédica no Brasil. Comparações com o desempenho científico brasileiro e mundial. Ciência \& Saúde Coletiva, 2004, 9 (3):303-327.

26. Brasil. Ministério da Saúde. Secretaria de Ciência, Tecnologia e Insumos Estratégicos. As ações de CTI em Saúde nos Sistemas Estaduais: PPSUS 2012. Fórum Nacional: Consecti-Confap. Curitiba 8 e 9 de março de 2012. Disponível em <www.confp.org.br/palestra/14hs_carlos_gadelha.ppt.> Acesso em: 05 dez 2014.

Artigo apresentado em 11-12-14

Artigo aprovado em 10-03-15 Artigo publicado no sistema em 22-12-15 